\title{
Production and Amyloid fibril formation of tandem repeats of recombinant Yeast Prion like protein fragment
}

\author{
Yongae Kim, Jae-Joon Park, Jung-Hyun Hwang, and Tae-Joon Park \\ Department of Chemistry, Hankuk University of Foreign Studies, Yongin, 449-791, Republic of \\ Korea \\ (Received Nov 30, 2011; revised December 07, 2011; accepted December 13, 2011)
}

\begin{abstract}
Amyloid fibrils have long been known to be the well known $\alpha$-helix to $\beta$ sheet transition characterizing the conversion of cellular to scrapie forms of the prion protein. A very short sequence of Yeast prion-like protein, GNNQQNY (SupN), is responsible for aggregation that induces diseases. KSI-fused tandem repeats of SupN vector are constructed and used to express SupN peptide in Escherichia coli (E.Coli). A method for a production, purification, and cleavage of tandem repeats of recombinant isotopically enriched SupN in E. coli is described. This method yields as much as $20 \mathrm{mg} / \mathrm{L}$ of isotope-enriched fusion proteins in minimal media. Synthetic SupN peptides and ${ }^{13} \mathrm{C}$ Gly labeled SupN peptides are studied by Congo Red staining, Birefringence and transmission electron microscopy to characterize amyloid fibril formation. To get a better understanding of aggregation-structure relationship of 7 residues of Yeast prion-like protein, the change of a conformational structure will be studied by ${ }^{13} \mathrm{C}$ solid-state $\mathrm{nmr}$ spectroscopy as powder of both amorphous and fibrillar forms.
\end{abstract}

Keywords : Amyloid, fibril, tandem repeats, Prion like protein

\section{INTRODUCTION}

A group of diseases are induced where proteins or fragments of proteins convert from their normally soluble forms to insoluble fibrils or plaques, which accumulate in a variety of organs including the liver, spleen, and brain. The final forms of these aggregates often have a well-defined fibrillar nature, and known as amyloid. ${ }^{1-4}$ A partial list includes alzheimer's diseases, parkinson's

* To whom correspondence should be addressed. E-mail : yakim@hufs.ac.kr 
176 Production and Amyloid Fibril Formation of Tandem Repeats of SupN

diseases, the spongiform encephalopathies such as Creutzfeldt-Jakob disease, type II diabetes, fatal familial insomnia. The range of proteins involved in these diseases is such as lysozyme, transthyretin and the prions.

A convenient model for studying both amyloid formation and the prion-like transmission of protein conformation is the yeast protein Sup35. Sup35 protein has some properties typical of mammalian prions, such as aggregation and resistance to protease. $^{1-2}$ Since a short peptide (SupN) from $\mathrm{N}$ terminus is very critical for maintenance of the phenotype, we expressed and reveal the molecular level of aggregation of SupN.

\section{EXPERIMENTAL}

Gene construction -fused-SupN multimer inserted pET31b(+) vector were constructed as below. Oligonucleotides for peptide SupN-Met-SupN-Met were 5'-GGT AAT AAC CAA CAG AAT TAC ATG GGT AAT AAC CAA CAG AAT TAC ATG-3' (forward sequence) and 5'-GTA ATT CTG TTG GTT ATT ACC CAT GTA ATT CTG TTG GTT ATT ACC CAT-3' (reverse sequence). Annealed oligonucleotides were purified by agarose gel electrophoresis and extracted using Gel Extraction Kit (Bioneer, Korea). Purified oligonucleotides were ligated with AlwN I digested dephosphorylated pET-31b(+)vector (Novagen, USA). Ligated mixture was transformed to a nonexpressed NovaBlue strain of E.coli. (Novagen, USA). The number (n) of SupN dimer units of the 
resulting clones was ascertained by agarose gel electrophoresis. The appropriate vectors were then transformed into BLR(DE3)pLysS (Novagen, USA) for overproduction. All KSI-fused genes were sequenced to verify mutations and the number of multiple SupN units.

Expression and purification of the fusion proteins with tandem repeats of supN - All proteins with KSI fused (SupN)n His-tags were expressed in Escherichia coli BLR(DE3)pLysS cells. Production of the KSI-(SupN) $)_{2,4,6}$-His-tags fusion protein was performed in M9 minimal medium. ${ }^{13} \mathrm{C}$ Glucose and $\left({ }^{15} \mathrm{NH}_{4}\right)_{2} \mathrm{SO}_{4}$ were used for preparation of uniformly ${ }^{13} \mathrm{C}$ and uniformly ${ }^{15} \mathrm{~N}$ enriched peptide SupN separately. When the $\mathrm{OD}_{600}$ is at 0.5 , transcription of the gene was induced by the addition of $1 \mathrm{mM}$ IPTG. The cells were harvested 4 to 5 hours after the induction when $\mathrm{OD}_{600}$ is decreased. Cells were suspened in the lysis buffer (20 mM Tris $\mathrm{HCl}, 500 \mathrm{mM} \mathrm{NaCl}, 5 \mathrm{mM}$ imidazole, $\mathrm{pH}$ 7.9 8.0) and cells and DNA fragmented by sonication (Sonics, 500W). Cell lysate was pelleted for 20 min at 12,000 rpm at $4{ }^{\circ} \mathrm{C}$ and pellet of inclusion body was redissolved in the lysis buffer with $6 \mathrm{M}$ Guanidine $\mathrm{HCl}$ for overnight at room temperature. After centrifugation for $30 \mathrm{~min}$ at 12,000 rpm at $4{ }^{\circ} \mathrm{C}$, the supernatant was loaded onto $\mathrm{Ni}^{2+}$-NTA-His Bind Resins (Novagen, USA). Column had been charged with $50 \mathrm{mM} \mathrm{NiSO}_{4}$ and equilibrated buffer containing $20 \mathrm{mM}$ Tris.HCl, $500 \mathrm{mM} \mathrm{NaCl}$, 5 mM imidazole, pH 7.9 8.0 with 6 M Guanidine-HCl. The column was washed with 5 column volumns of $20 \mathrm{mM}$ Tris.HCl, $500 \mathrm{mM} \mathrm{NaCl}, 16 \mathrm{mM}$ imidazole, $\mathrm{pH}$ 7.9 8.0 with $6 \mathrm{M}$ Guanidine-HCl. KSI-fused (SupN)n His-tags was eluted with $20 \mathrm{mM}$ Tris.HCl, $500 \mathrm{mM} \mathrm{NaCl,} 500 \mathrm{mM}$ Imidazole, 
178 Production and Amyloid Fibril Formation of Tandem Repeats of SupN

pH 7.9 8.0 with $6 \mathrm{M}$ Guanidine-HCl. Eluted fusion peptides were dialyzed overnight against $\mathrm{H}_{2} \mathrm{O}$ in 10 kDa MWCO dialysis bag. White precipitate from dialysis was lyophilized and dissolved into 70\% formic acid. Prestabilized $\mathrm{CNBr}$ at room temperature was added to the sample solution at 100 -fold molar excess over all methionyl residues in the fusion protein. The flask was wrapped in aluminum foil and incubated for at least 3 hrs at room temperature. Reaction products were 10 fold diluted with

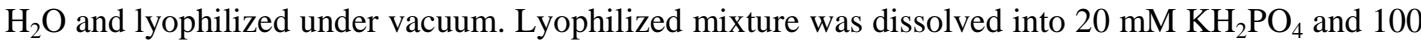
mM NaCl, pH 7.4 and centrifuged at $5000 \mathrm{~g}$ for $1 \mathrm{hr}$ at $4{ }^{\circ} \mathrm{C}$. Soluble peptide SupN in the Supernatant was purified by RP-HPLC on a Waters Delta-Pak C18 column (7.8x300 mm ID). Elution was carried out at room temperature with a linear gradient from $0 \%$ to $100 \%$ of buffer B over 60 mins at a flow rate of $3 \mathrm{ml} / \mathrm{min}$ with the absorbance monitored at $220 \mathrm{~nm}$ and $280 \mathrm{~nm}$ using a DIONEX P580. Buffer A was 5\% acetonitrile and $0.1 \%$ TFA in $\mathrm{H}_{2} \mathrm{O}$ and buffer B was $95 \%$ acetonitrile and $0.1 \%$ TFA in water. The resultant SupN peptide fractions were collected and dried under vacuum in a lyophilizer (Labconco, USA). Peptide concentrations were determined by measuring absorbance at $276 \mathrm{~nm}$. Identity of peptide was confirmed by ESI or MALDI mass spectrometry.

Fibrillization of GNNQQNY(M) peptide from KSI-fused (SupN) $)_{n}$ His6 peptide -Lyophilized SupN peptide was dissolved in $20 \mathrm{mM} \mathrm{KH}_{2} \mathrm{PO}_{4}$ and $100 \mathrm{mM} \mathrm{NaCl}, \mathrm{pH} 7.4$ to a final concentration of 5-10 mg/ml. GNNQQNY peptides were fibrillized by incubation of the peptide solution for 5-30 days at $24{ }^{\circ} \mathrm{C}$. Fibrillized solutions were lyophilized for NMR measurements. Synthesized peptides which 
were performed by Anygen, Inc. (Daejeon, Korea) were also fibrillized. The correct identity of the synthetic peptide was confirmed by ion spray mass spectrometry, and the purity of the peptides was confirmed by reverse phase high pressure liquid chromatography. Various conditions were used to obtain fiber. The best results were obtained as below. The peptides were dissolved in DMSO, and then diluted with buffer (0.02 M NaCl/0.01 M Tris, pH 7.2) to a final concentration of approximately $2 \mathrm{mM}$. And peptide were incubated at room temperature for $7 \sim 13$ days.

Congo Red staining and Birefringence - Birefringence was determined using the same solution of peptides that were used for fibrillization experiments. A $10 \mu$ aliquot of the suspension of peptide aged for 10 days was allowed to dry on a glass microscope slide. Staining was performed by addition of a solution of $80 \%$ ethanol saturated with Congo Red and $\mathrm{NaCl}$. Birefringence was determined with an Axioskop-40 (Carl Zeiss Inc.) stereoscope equipped with a polarizing stage.

Transmission Electron Microscope(TEM) -A drop $(\sim 10 \mu \ell)$ of fibrillized peptide solution is applied to a carbon-coated film on a 200-mesh copper grid. After 1 min, excess fluid was removed, and the grid was then negatively stained with $2 \%$ uranyl acetate in water and dried for $30 \mathrm{~min}$. Samples were viewed on a Jeol JEM 1010 transmission electron microscope at an accelerating voltage of $80 \mathrm{kV}$ program. 
180 Production and Amyloid Fibril Formation of Tandem Repeats of SupN

\section{RESULTS AND DISCUSSION}

Gene construction The overall strategy for construction and production of the SupN proteins is shown in Figure $1 .^{5-6}$ Complementary oligonucleotides encoding SupN peptide-Met-SupN peptideMet were synthesized, annealed, and unidirectionally self-ligated using three-base-ATG-3', 3'-TAC-. These cohesive ends form tandem repeated SupN units spaced by single methionine codons, ATG.

Construct of pET31b(+) with SupN tandem inserts was confirmed by a $1.5 \%$ agarose gel electrophoresis after double digested with Xba1 and XhoI of purified plasmid DNA in Figure 2(a).

Figure 2(a, lane 3, 4, and 5) shows corresponding plasmid DNA inserts of SupN tandem inserts of $n=1,2$, 3. Each of the different KSI-(SupNM-SupNM)n-His6 (n=1, 2, and 3) were expressed in the protease deficient strain BLR(DE3)pLysS successfully. The production levels are shown in Figure 2(b). 


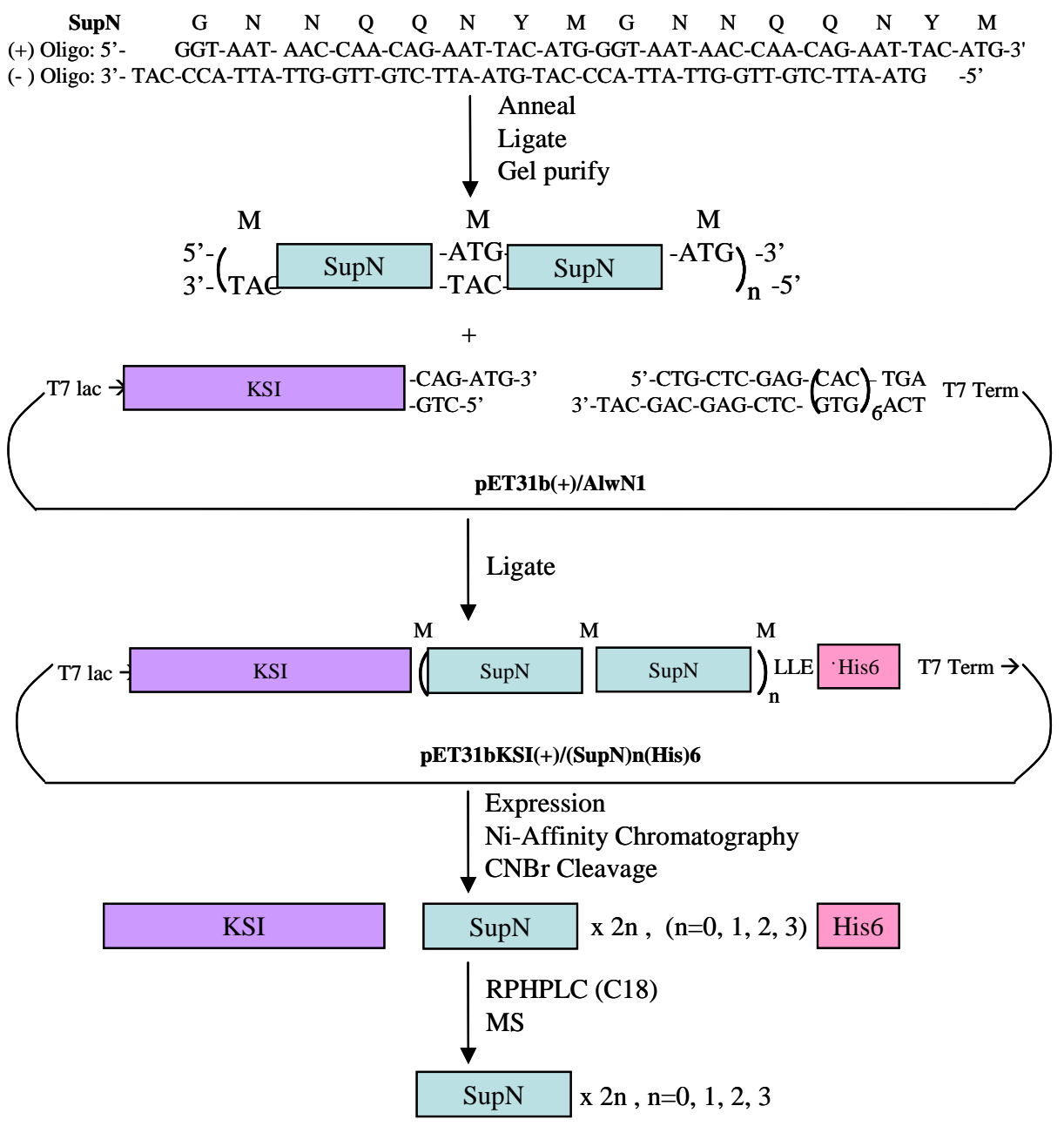

Figure 1. Schematic representation for construction, production, purification of Ketosteroid isomerase(KSI)-multiple supN peptide-His6 fused proteins. Oligonucleotides encoding both strands of the 7-13 amino acids of Sup35 were annealed, unidirectionally self-ligated to form an array of tandem repeats of SupN separated by ATG codons. These multimers(n=1, 2, and 3) were then lagated into the AlwNI site of pET31b(+) to make KSI-(supN-M-supN-M)n-His6 fusion genes where $n=0,1$, 2, 3. Expression levels were tested by SDS-PAGE, the highest yielding fusion protein( $\mathrm{n}=3$ ) was purified by Ni-affinity chromatography and cleaved by CNBr into supN, KSI, and His6. SupN was isolated by C18 reverse phase HPLC and checked by ESI MS. 

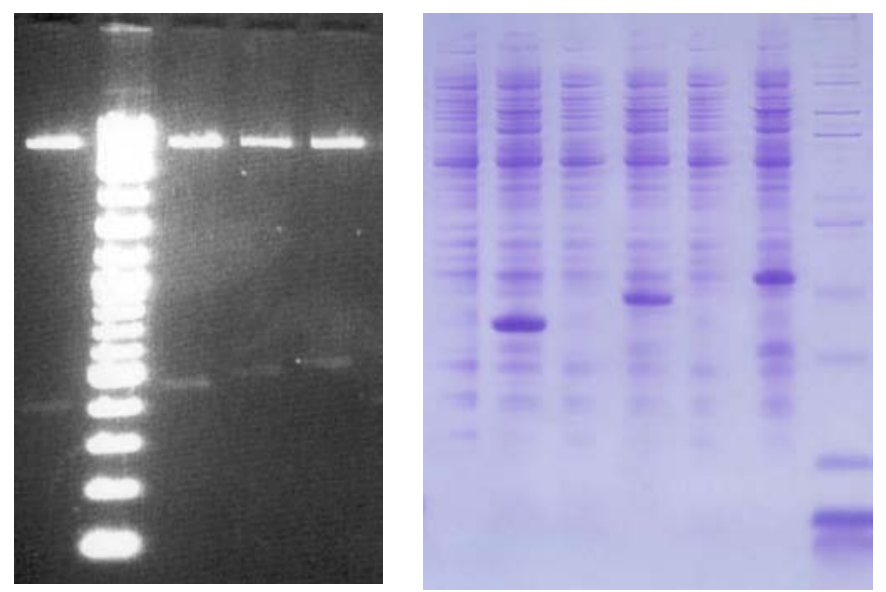

Figure 2. $1.5 \%$ agarose gel electrophoresis after double digested with Xba1 and XhoI of purified plasmid DNA and production of KSI fused SupN with tandem repeats in E. coli by 12\% SDS-PAGE. (a) lane1, empty vector control (423 bp); lane 2, DNA ladder 100, 200, 300bp, ... ; lane 3, 4, and 5 shows corresponding plasmid DNA inserts of SupN tandem inserts of n=1(48 bp), 2(96 bp), 3(144 bp). (b) Lane 2, 4, and 6 are insoluble protein fractions from IPTG induced BLR(DE3)pLysS where $\mathrm{n}=1,2$, and 3. Lane 1, 3, and 5 are protein fractions before induction. Molecular weight of KSI is about $14 \mathrm{kDa}$. Molecular weight of SupN dimmer is about $2 \mathrm{kDa}$.
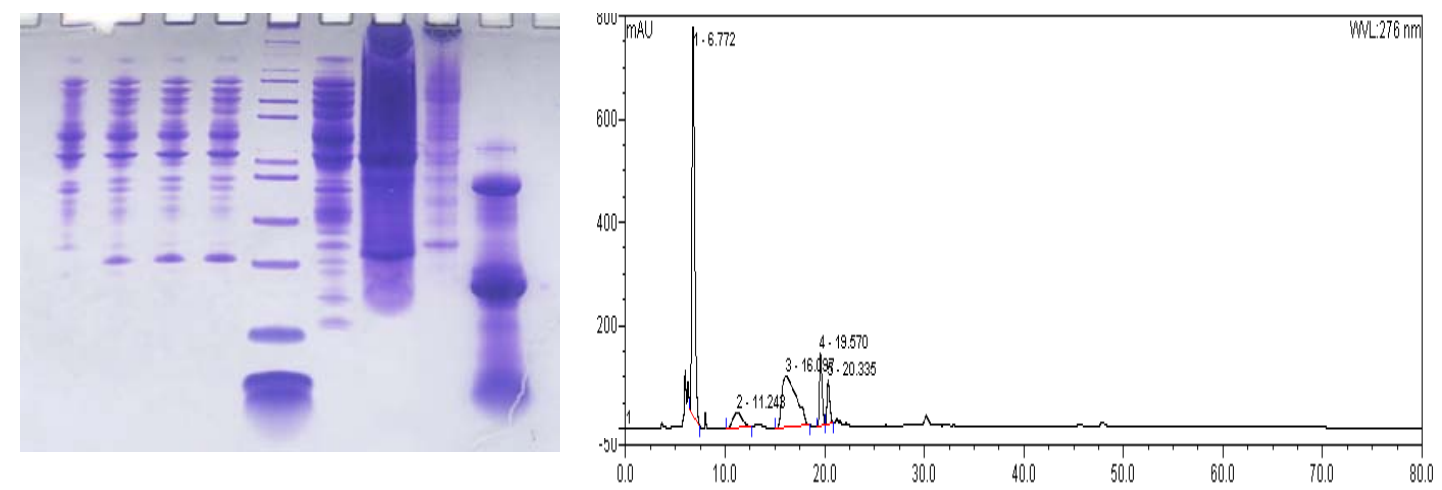

Figure 3. Coomassie blue stained (a) 12\% tris-tricine PAGE showing samples from expression and Ni-NTA column purification of the uniformly ${ }^{13} \mathrm{C}$ labeled KSI fusion protein. Lane 1, cells before IPTG induction; Lane 2, cells after 1 hr IPTG induction; Lane 3, cells after 2 hrs IPTG induction; 
Lane 4, cells after 3 hrs IPTG induction; Lane 5, Marker; Lane 6, soluble fraction after lysis; Lane 7, insoluble fraction after lysis; Lane 8, KSI-fused protein purified from a Ni-NTA affinity chromatography; Lane 9, reaction mixtures after CNBr cleavage. (b) Reversed phase HPLC chromatogram. Peptides were eluted with a linear gradient of ACN in water containing $0.1 \%$ TFA on the C4 column. Eluent A: $95 \% \mathrm{ddH}_{2} \mathrm{O}$ with 0.1\% TFA and 5\% ACN, Eluent B: $5 \%$ ddH2O with $0.1 \%$ TFA and $95 \%$ ACN.

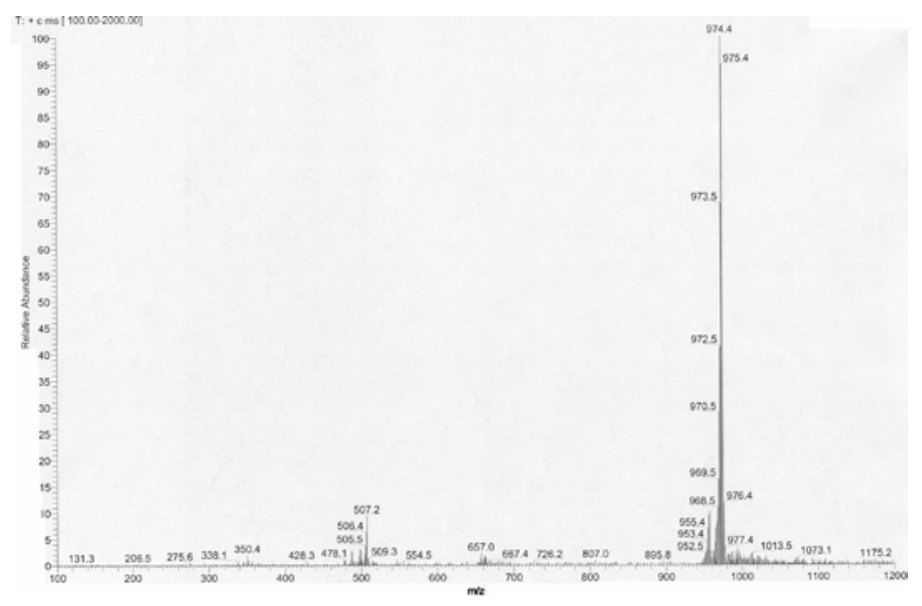

Figure 3.Electrospray Mass spectra of SupN peptide. Uniformly ${ }^{13} \mathrm{C}$ labeled GNNQQNYM, SupN peptide was expressed in E. coli. Major free acid form of 975 Da and minor homoserine lactone form of 957 Da are shown.

\section{Fibrillization of GNNQQNY(M) peptide from KSI-fused (SupN) ${ }_{n}$-His6 peptide -}

Identification of Fibril-forming - The fibrillization potential of peptide fragments was examined by Congo Red Staining, Birefringence, and TEM(Transmission Electron Microscope). ${ }^{7-11}$ Two fibrillar structures of synthetic and expressed peptides were obtained (Figure 4 a and b) by TEM. Green-gold birefringence after staining with Congo Red is one of the best characteristics of amyloid fibrils. The 
184 Production and Amyloid Fibril Formation of Tandem Repeats of SupN

studied peptides have showed some degree of birefringence. The green birefringence that was observed with the synthetic and expressed peptides was clear and strong. Fibrillar structures are similar to other amyloid fibrils which reported paper by other workers. ${ }^{7-9}$ The expressed peptides were shown better fibrillar structures than that of synthetic peptides.
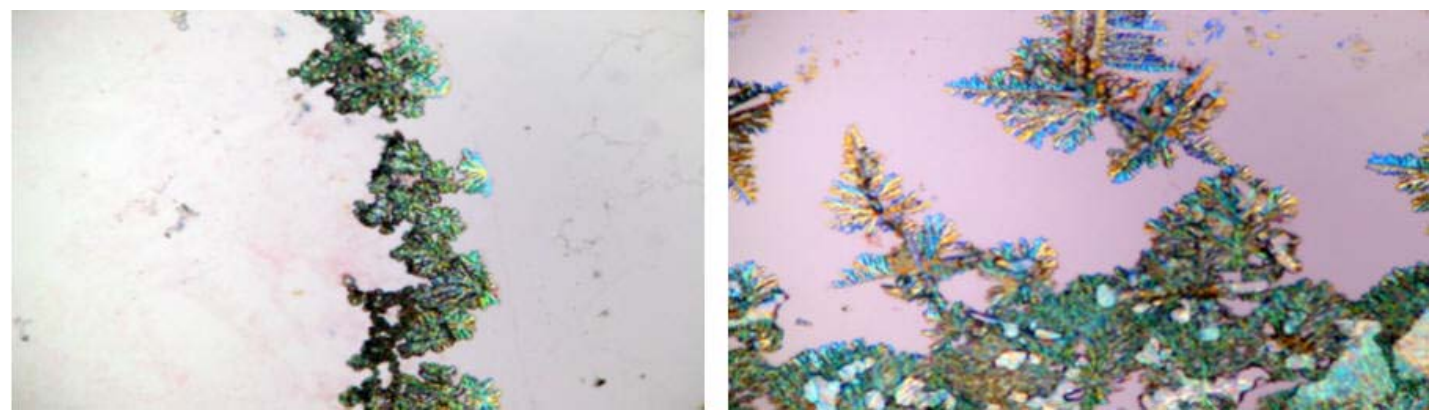

Figure 4. Green-gold birefringence of fibrillized synthetic SupN (a) and uniformly ${ }^{13} \mathrm{C}$ labeled expressed SupN (b) was measured with Polarizing Microscope: asioskop 40 (x100, Zeiss)

Transmission Electron Microscopy (TEM) TEM images were observed using the TEM visualization to further determine the properties of the fibrillar structure in figure 5. Expressed SupN were well observed more than synthetic SupN at fibrillar state. And fibrillization of peptide was confirmed by TEM. Therefore the sample solutions were used because it was studied conformation of fibrillar state SupN. 


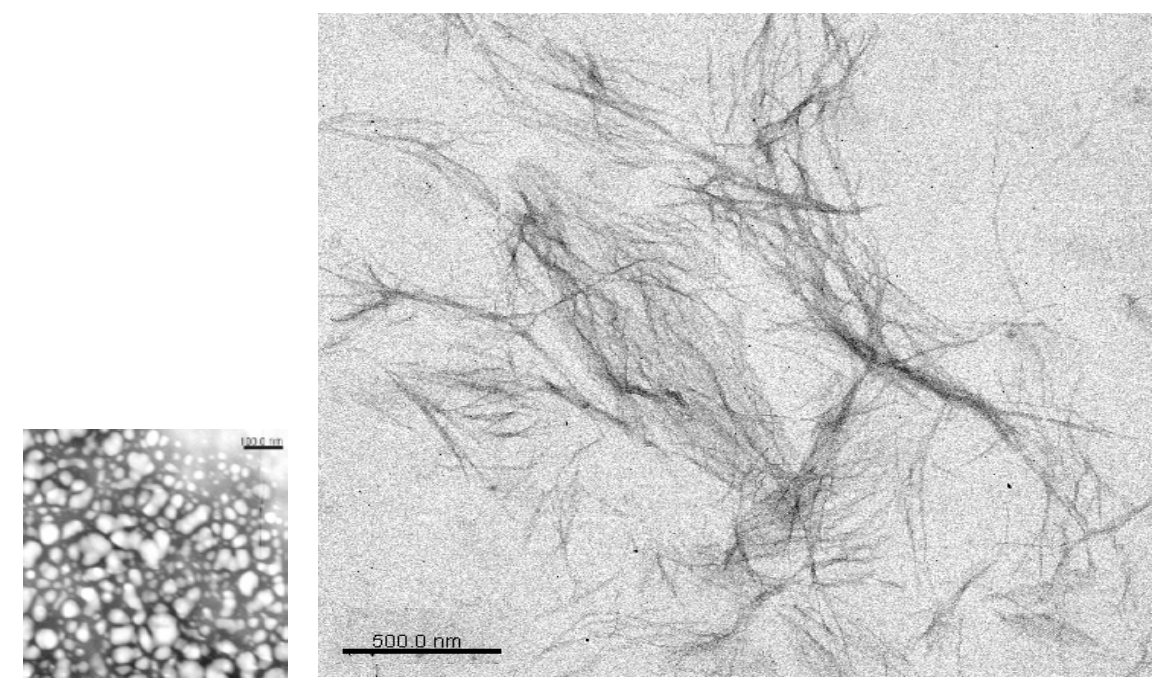

Figure 5. TEM images of amorphous aggregates (a) and insoluble fibrillar states (b) in an aged solution of SupN peptide. Scale bar represents 100nm.

\section{CONCLUSION}

In this paper, we demonstrate the optimization of expression, purification, and fibrillization of SupN peptides. Milligram quantities of SupN peptides were obtained by using preparative reversedphase HPLC. Purified SupN peptides formed as a fibrillar state under incubating condition. Synthetic SupN peptides of unlabeled and expressed SupN peptides of ${ }^{13} \mathrm{C}$ Gly labeled were studied with Congo Red staining and Birefringence and Transmission Electron Microscopy that are used to characterize amyloid fibril formation. To get a better understanding of aggregation-structure relationship of 7 residues of Yeast prion-like protein, the change of a conformational structure will soon be studied by ${ }^{13} \mathrm{C}$ solid-state nmr spectroscopy as powder of both amorphous and fibrillar forms. 
186 Production and Amyloid Fibril Formation of Tandem Repeats of SupN

\section{Acknowledgments}

This work was supported by a Hankuk University of Foreign Studies Research Fund of 2011.

\section{REFERENCES}

1. J.W. Kelly, Curr. Opi. Struct. Biol. 8, 101, (1998).

2. C.M. Dobson, Trends Biochem. Sci. 24, 329, (1999).

3. R.B. Wickner, Science 264, 566, (1994).

4. H. E. Sparrer, A. Santoso, F.C. Szoka, J.S. Weissman Science 289, 595, (2000).

5. T. J. Park, S. Y. Im, J. S. Kim, Y. Kim, Proc. Biochem. 45, 682, (2010).

6. J. S. Kim, T. J. Park, Y. Kim, J. Kor. Magn. Reson. 13, 96, (2009).

7. M. Reaches, Y. Porant, E. Gazit, J. Biol. Chem. 277, 35475, (2002).

8. W. Zou, D. Sheng, P. X. Fraser, N. R. Cashman, A. Chakrabartty, Eur. J. Biochem. 268, 4885, (2001).

9. R. Khurana, V. N. Uversky, L. Nielson, A. L. Fink, J. Biol. Chem. 276, 22715, (2001).

10. X. Wu and K. W. Zilm, J. Mag. Res. A 111, 29, (1994).

11. O.N. Antzutkin, R. Tycko, J. Chem. Phys. 110 , 2749, (1999). 